\title{
Is Bone Grafting Necessary in Opening Wedge High Tibial Osteotomy? A Meta-Analysis of Radiological Outcomes
}

\author{
Jae Hwi Han, $\mathrm{MD}^{1}$, Hyun Jung Kim, $\mathrm{PhD}^{2}$, Jae Gwang Song, $\mathrm{MD}^{1}$, Jae Hyuk Yang, $\mathrm{MD}^{3}$, Nikhl N Bhandare, \\ $\mathrm{MD}^{4}$, Aldrich Raymund Fernandez, $\mathrm{MD}^{5}$, Hyung Jun Park, $\mathrm{MD}^{1}$, and Kyung Wook Nha, $\mathrm{MD}^{1}$ \\ ${ }^{1}$ Department of Orthopaedic Surgery, Inje University Ilsan Paik Hospital, Goyang; ${ }^{2}$ Department of Preventive Medicine, Institute for Evidence-based Medicine, Korea \\ University College of Medicine, Seoul; ${ }^{3}$ Department of Orthopaedic Surgery, Veterans Health Service Medical Center, Seoul, Korea; ${ }^{4}$ Department of Orthopaedic \\ Surgery, Bhandare Hospital, Panaji, India; ${ }^{5}$ Department of Orthopaedic Surgery, Army General Hospital, Bonifacio, Philippines
}

\begin{abstract}
Purpose: Bone grafting in opening wedge high tibial osteotomy (OWHTO) is still controversial. The purpose of this study is to compare the radiological outcomes of OWHTO with bone graft (autogenous, allogenous, and synthetic bone graft) and those without bone graft.

Materials and Methods: PubMed, MEDLINE, EMBASE and Cochrane Register of Studies databases were searched using specific inclusion and exclusion criteria for radiological studies involving OWHTO with bone graft and without bone graft groups. All reported delayed union, nonunion and correction loss were analyzed. Data were searched from the time period of January 2000 through July 2014. In addition, a modified Coleman methodology score (CMS) system was used to assess the methodological quality of the included studies.

Results: Twenty-five studies with a mean CMS value of 77 (range, 61 to 85 score) were included. In total, 1,841 patients underwent OWHTO using 4 different procedures for bone graft: autobone graft $(n=352)$, allobone graft $(n=547)$, synthetic bone graft $(n=541)$ and no bone graft $(n=401)$. There was a similar tendency for delayed union, nonunion and correction loss rate among the osteotomy space filling methods.

Conclusions: The meta-analysis showed there was a similar tendency for radiological union and correction maintenance among patients undergoing OWHTO regardless of the type of bone in all of the studies. However, the currently available evidence is not sufficient to strongly support the superiority of OWHTO with bone graft to OWHTO without bone graft.
\end{abstract}

Keywords: Knee, Osteoarthritis, Osteotomy, Bone graft, Meta-analysis

\section{Introduction}

Opening wedge high tibial osteotomy (OWHTO) with medial plate fixation is a proven adequate and safe method for treatment of medial compartment osteoarthritis of the knee and varus deformity, particularly in young and/or active individuals ${ }^{1,2)}$.

Received September 21, 2014; Revised (1st) May 10, 2015;

(2nd) September 14, 2015; Accepted September 17, 2015

Correspondence to: Kyung-Wook Nha, MD

Department of Orthopaedic Surgery, Inje University Ilsan Paik Hospital, 170 Juhwa-ro, Ilsanseo-gu, Goyang 10380, Korea

Tel: +82-31-910-7312, Fax: +82-31-910-7319

E-mail:kwnhamj@hotmail.com

This is an Open Access article distributed under the terms of the Creative Commons Attribution Non-Commercial License (http://creativecommons.org/licenses/by-nc/4.0/) which permits unrestricted non-commercial use, distribution, and reproduction in any medium, provided the original work is properly cited.
Advantages of OWHTO, compared to closed wedge osteotomy, include preservation of bone stock, predictable and adjustable correction, relatively easy exposure with avoidance of proximal tibio-fibular joint disruption, fibular nerve palsy, and compartment syndrome ${ }^{3)}$.

However, OWHTO creates a gap in the metaphysis of the tibia. To enhance bone healing and increase initial mechanical stability, a high degree OWHTO may require the application of bone graft/substitute to fill the osteotomy gap. Moreover, OWHTO has been associated with risk of nonunion, collapse and loss of correction $^{4)}$. Autologous bone graft is the "gold standard" to fill the bone defect, but iliac crest graft harvest has risks. The associated complications include pain, thigh hypoesthesia, infection, pelvic bone fracture and discomfort wearing clothes ${ }^{5,6)}$. Allograft to avoid donor side morbidity was successfully applied in $\mathrm{HTO}^{7}$. However, the use of allograft increases additional risks such as disease transmission, immunologic reactions and slow remodeling. 
Due to the limited autologous bone availability and the problem of donor-site morbidity, many efforts have been made to find adequate supporting material for augmentation after osteotomy. Hydroxyapatite, $\beta$-tricalcium phosphate or the combination of both are the most commonly used synthetic augments in $\mathrm{OWH}$ TO. In the meantime, some recent studies presented good shortterm results of OWHTO without additional bone substitutes for filling the osteotomy gap ${ }^{8,9)}$. Still, the most suitable material for filling the opening space in OWHTO has not been identified.

Over the past decade, there has been only one randomized controlled study on the comparison of autologous bone grafting and no filling method, which reported similar outcomes between the two methods ${ }^{10)}$. Although randomized controlled trials (RCTs) are considered to offer the ideal and highest level of evidence for patient care, numerous "good" surgical practices have evolved into the "standard of care" without being randomized against placebo or ineffective treatment options ${ }^{11)}$, which probably explains why only one RCT has been published. Considering that observational studies constitute the best available evidence ${ }^{12)}$, we attempted to conduct a meta-analysis of observational studies for the purpose of providing surgeons with informed data for better decision-making in OWHTO.

Table 1. Inclusion and Exclusion Criteria

\begin{tabular}{|c|c|}
\hline Inclusion criteria & Exclusion criteria \\
\hline Studies on patients who received OWHTO & Studies on patients who received closing wedge HTO \\
\hline $\begin{array}{l}\text { Studies reporting minimum 1-year follow-up data on radiological } \\
\text { outcomes and complications of OWHTO }\end{array}$ & $\begin{array}{l}\text { Studies reporting less than 1-year follow-up data on radiological } \\
\text { outcomes and complications of OWHTO }\end{array}$ \\
\hline Level I, II, III, or IV evidence & $\begin{array}{l}\text { Level V evidence (case report, technical note, letter to editor), } \\
\text { biomechanical reports, and review articles }\end{array}$ \\
\hline Articles written in English & Articles written in languages other than English \\
\hline Human subjects & Non-human subjects \\
\hline $\begin{array}{l}\text { Study published or in press online between January 1, } 2000 \text { and May } \\
1,2014\end{array}$ & Study published or in press online on or before January 1,2000 \\
\hline Medial plate fixation for OWHTO & Other devices (external fixator, staple) for OWHTO \\
\hline
\end{tabular}

OWHTO: opening wedge high tibial osteotomy.

Table 2. Search Protocol

\begin{tabular}{|c|c|c|}
\hline No. & Search terms & Results \\
\hline 1 & osteoarthritis [tiab] & 35,633 \\
\hline 2 & "osteoarthritis" [MeSH:NoExp] & 28,000 \\
\hline 3 & $1 \mathrm{OR} 2$ & 49,340 \\
\hline 4 & "knee joints" [tiab] OR knee [tiab] OR tibias [tiab] OR tibia [tiab] & 106,972 \\
\hline 5 & ("knee" [MeSH]) OR "knee joint" [MeSH]) OR " tibia" [MeSH] & 72,058 \\
\hline 6 & 4 OR 5 & 134,573 \\
\hline 7 & 3 AND 6 & 16,821 \\
\hline 8 & $\begin{array}{l}\text { "osteoarthritis, knee" [MeSH] OR "genu varum" [MeSH] OR "tibia/radiography" [MeSH] OR "tibia/surgery" [MeSH] } \\
\text { OR "tibia/therapy" [MeSH] OR "genu valgum" [MeSH] }\end{array}$ & 20,120 \\
\hline 9 & $\begin{array}{l}\text { "knee osteoarthritis" [tiab] OR "osteoarthritis of knees" [tiab] OR "osteoarthritis of knee" [tiab] OR "genu varum" } \\
\text { [tiab] OR "medial gonarthrosis" [tiab] OR "valgus knee" [tiab] OR "varus knee" [tiab] OR "varus deformity" [tiab] } \\
\text { OR "valgus deformity" [tiab] OR "genu valgum" [tiab] }\end{array}$ & 7,381 \\
\hline 10 & 8 OR 9 & 23,707 \\
\hline 11 & 7 OR 10 & 31,784 \\
\hline 12 & "osteotomy" [MeSH:NoExp] OR osteotomy [tiab] OR osteotomies [tiab] & 31,896 \\
\hline 13 & 11 AND 12 & 3,664 \\
\hline 14 & 13 AND publication date from $2000 / 01 / 01$ to $2014 / 05 / 02$ & 2,057 \\
\hline
\end{tabular}


The purpose of this study was to compare the radiological outcomes of OWHTO with bone graft (autogenous graft, allogenous graft, and synthetic bone) and those without bone graft. The hypotheses were that the use of bone graft would produce superior radiological outcomes.

\section{Materials and Methods}

\section{Eligibility Criteria}

Published studies were included in this meta-analysis if they met the eligibility criteria described in Table 1. Patients included in this study were limited to osteoarthritis patients. There was no limitation in the age of patients. Fixation method was limited to plate fixation using either a locking or a non-locking plate.

\section{Data Sources and Search Strategy}

A literature search of online databases (MEDLINE, EMBASE and Cochrane Register of Studies) was performed. The search strategy used in the case of MEDLINE is presented in Table 2, which was modified for each of the other databases (Table 2).

Next, the references from the included studies were screened, and experts in the field were contacted for help in identifying additional studies. Two independent review authors (Han and Song) selected citations each based on the titles and abstracts. The eligibility of the full papers of those citations initially thought to fulfill the inclusion criteria was then assessed. In cases where a consensus could not be reached, a third review author (Nha) was consulted.

Table 3. Criteria of the Coleman Methodology Score for Studies

\begin{tabular}{|c|c|c|c|}
\hline Criteria & Score & Criteria & Score \\
\hline Part A-only one score to be given for each of the seven sections & & 6. Description of surgical procedure & \\
\hline 1. Study size-number of otsteotomies & & Adequate (technique stated with necessary details of the & 5 \\
\hline$>60$ & 10 & type of procedure) & \\
\hline $41-60$ & 7 & Fair (technique only stated without elaboration) & 3 \\
\hline $20-40$ & 4 & Inadequate, not stated or unclear & 0 \\
\hline 2. Mean follow-up (mo) & & 7. Description of postoperative rehabilitation & \\
\hline$>24$ & 5 & Well described with $>80 \%$ of patients complying & 10 \\
\hline $12-24$ & 2 & Well described with $60 \%-80 \%$ of patients complying & 5 \\
\hline$>12$, not stated or unclear & 0 & Protocol not reported or $<60 \%-80 \%$ of patients complying & 0 \\
\hline $\begin{array}{l}\text { 3. Number of different surgical procedures included in each } \\
\text { reported outcome }\end{array}$ & & $\begin{array}{l}\text { Part B-scores may be given for each option in each of the three } \\
\text { sections if applicable }\end{array}$ & \\
\hline One surgical procedure only & 10 & 1. Outcome criteria & \\
\hline More than one surgical procedure, but $>90 \%$ of subjects & 7 & Outcome measures clearly defined & 2 \\
\hline undergoing one procedure & & Timing of outcomes assessment clearly stated & 2 \\
\hline Not stated, unclear or $<90 \%$ of subjects undergoing one & 0 & Use of outcome criteria with good reliability & 3 \\
\hline procedure & & Use of outcome with good sensitivity & 3 \\
\hline 4. Type of study & & 2. Procedure for assessing outcomes & \\
\hline Randomised control trial & 15 & Subjects recruited & 5 \\
\hline Prospective cohort study & 10 & Investigator independent of surgeon & 4 \\
\hline Retrospective cohort study & 0 & Written assessment & 3 \\
\hline 5. Diagnostic certainty & & Completion of assessment by subjects themselves with & 3 \\
\hline In all & 5 & minimal investigator assistance & \\
\hline In $>80 \%$ & 3 & 3. Description of subject selection process & \\
\hline \multirow[t]{3}{*}{ In $<80 \%$, no, unclear } & 0 & Selection criteria reported and unbiased & 5 \\
\hline & & Recruitment rate reported: $>80 \%$ or $<80 \%$ & 5 or 3 \\
\hline & & $\begin{array}{l}\text { Eligible subjects not included in the study satisfactorily } \\
\text { accounted for or } 100 \% \text { recruitment }\end{array}$ & 5 \\
\hline
\end{tabular}




\section{Data Abstraction}

A standardized form was used to extract data from the included papers on study characteristics, patient characteristics, surgical intervention, duration of follow-up, osteotomy size, postoperative rehabilitation protocol, radiological bone union period, nonunion rate, delayed union rate, correction loss rate and graftrelated problems. The extracted data were then cross-checked for accuracy. Disagreements were settled by the Nha.

\section{Statistical Analysis}

Statistical analysis was performed using STATA/MP ver. 13.0 (Stata Corp., College Station, TX, USA). Effect sizes for dichotomous data (incidence of nonunion, delayed union, and loss of correction) were calculated using random-effect models and expressed as event rates. The mean values of the continuous data (the mean bone union period; the mean difference in bone union period) were compared using a t-test. For each effect size, a 95\% confidence interval was given. Heterogeneity was calculated according to the method of Higgins et al. ${ }^{13)}$ and expressed as $\mathrm{I}_{2}$ (range, 0\% [complete consistency] to $100 \%$ [complete inconsistency]). Analysis of comparative studies and level IV case series analysis were performed separately. Case series analysis was performed to investigate whether the case series support the results of the one RCT and four comparative studies.

\section{Quality Assessment}

The methodological quality of the included studies was assessed by the two review authors (Han and Song) using the 10 critical appraisal criteria of the Coleman methodology score (CMS) (Table 3). The final scores ranged from 0 to 100 , with a perfect score (100) indicating a study design that completely avoids the influences of chances, various biases, and confounding factors.

\section{Included Studies}

Based on the full-text review, the final meta-analysis included 25 studies on OWHTO. A flowchart illustrating the study selection process is depicted in Fig. $1^{14)}$. The included studies are listed in Table 4. Twenty case series, one RCT and four comparative studies were included in the meta-analysis.

\section{Results}

\section{Quality Assessment}

The modified CMS value for individual study is presented in Table 3. The mean modified CMS value for all included studies was 77 (range, 61 to 85 score). The mean CMS value for each criterion is shown in Table 5 .

\section{Radiological Outcomes}

The overall results are shown in Table 6.

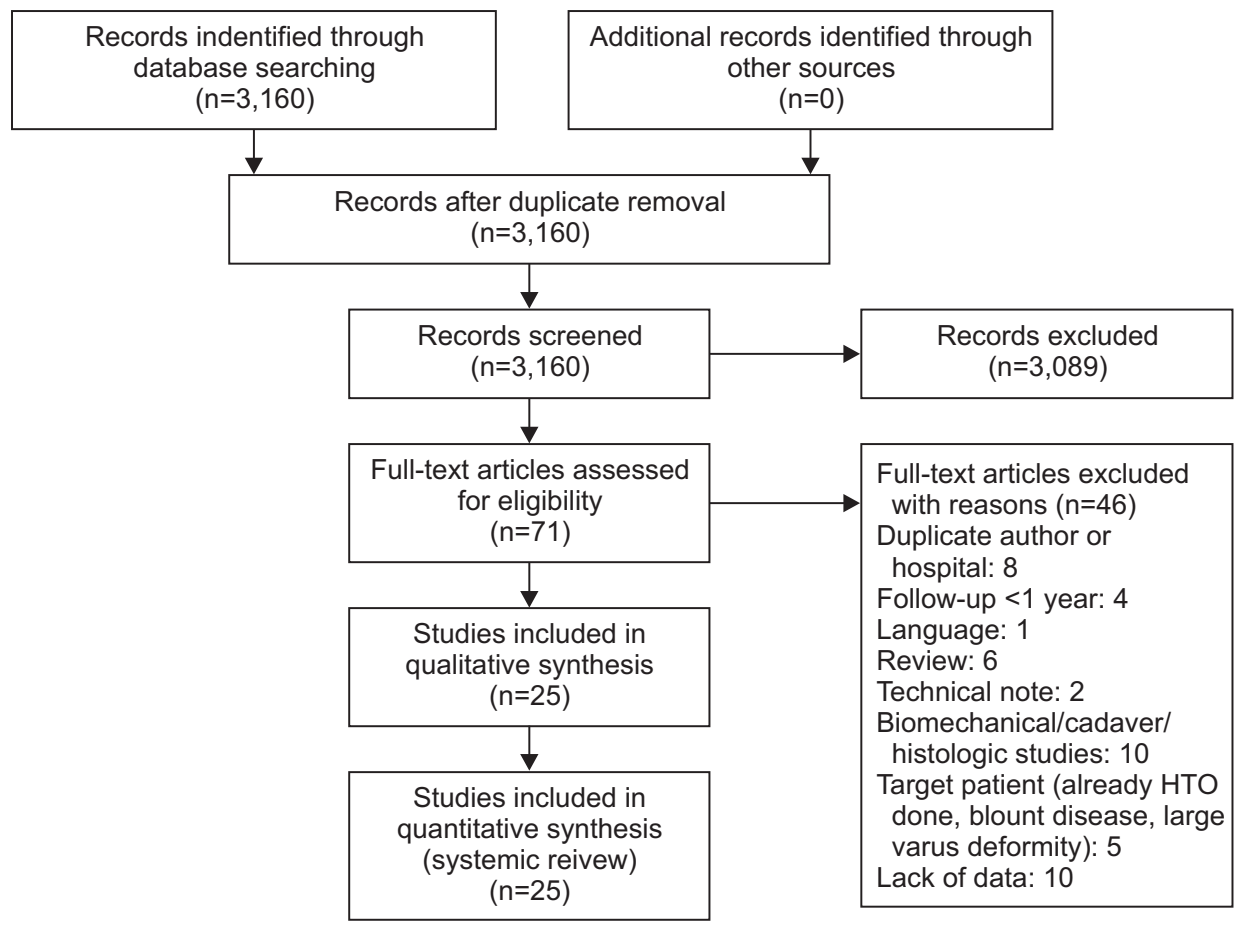

Fig. 1. Flowchart of identification of opening wedge high tibial osteotomy studies. HTO: high tibial osteotomy. Reprint from Moher et al. ${ }^{14)}$ 
Table 4. Studies Included in the Meta-Analysis

\begin{tabular}{|c|c|c|c|c|c|c|c|}
\hline Author & Year & $\begin{array}{c}\text { No. of } \\
\text { patients }\end{array}$ & Filler type & $\begin{array}{l}\text { Follow-up } \\
(\mathrm{mo})\end{array}$ & $\begin{array}{c}\text { Design/level of } \\
\text { evidence }\end{array}$ & Fixation type & CMS \\
\hline Chae et al. ${ }^{5)}$ & 2011 & 138 & Auto & 37 & Case/4 & T plate & 75 \\
\hline Yacobucci and Cocking ${ }^{7}$ & 2008 & 50 & Allo & 25 & Case $/ 4$ & Puddu plate & 79 \\
\hline \multirow[t]{2}{*}{ Zorzi et al. ${ }^{10)}$} & 2011 & 23 & Auto & 24 & $\mathrm{RCT} / 1$ & Puddu plate & 84 \\
\hline & 2011 & 23 & No & 24 & & Puddu plate & \\
\hline Bode et al. ${ }^{15)}$ & 2013 & 52 & No & 60 & Case/4 & TomoFix & 73 \\
\hline Schroter et al. ${ }^{16)}$ & 2011 & 35 & No & 12 & Case/4 & Aescula plate & 76 \\
\hline El-Azab et al. ${ }^{17)}$ & 2011 & 50 & No & 36 & Case $/ 4$ & TomoFix & 82 \\
\hline Brosset et al. ${ }^{18)}$ & 2011 & 51 & No & 24 & Case/4 & TomoFix & 72 \\
\hline El-Assal et al. ${ }^{19)}$ & 2010 & 59 & No & 38 & Case/4 & 4 holes toothed plate & 85 \\
\hline Kolb et al. ${ }^{20)}$ & 2009 & 51 & No & 52 & Case $/ 4$ & TomoFix & 85 \\
\hline Zaki and $\mathrm{Ra}^{21)}$ & 2009 & 50 & No & 60 & Case $/ 4$ & TomoFix & 75 \\
\hline Shim et al. ${ }^{22)}$ & 2013 & 37 & Auto & 36 & Case $/ 4$ & Arthrex plate & 61 \\
\hline Schroter et al. ${ }^{23)}$ & 2013 & 32 & Auto & 77 & Case/4 & LC-DCP & 82 \\
\hline Noyes et al. ${ }^{24)}$ & 2006 & 55 & Auto & 20 & Case/4 & Arthrex plate & 83 \\
\hline Haviv et al. ${ }^{25)}$ & 2012 & 22 & Allo & 75 & Case/4 & Puddu plate & 79 \\
\hline Santic et al. ${ }^{26)}$ & 2010 & 310 & Allo & 71 & Case/4 & T plate $(\mathrm{AO})$ & 83 \\
\hline DeMeo et al. ${ }^{27)}$ & 2010 & 20 & Allo & 100 & Case $/ 4$ & Puddu plate & 82 \\
\hline Saito et al. ${ }^{28)}$ & 2014 & 64 & Synthetic & 78 & Case/4 & TomoFix & 70 \\
\hline Saragaglia et al. ${ }^{29)}$ & 2011 & 124 & Synthetic & 125 & Case $/ 4$ & T plate $(\mathrm{AO})$ & 70 \\
\hline Ozalay et al. ${ }^{30)}$ & 2009 & 15 & Synthetic & 27 & Case/4 & Medial rigid angle plate & 80 \\
\hline Koshino et al. ${ }^{31)}$ & 2003 & 21 & Synthetic & 79 & Case $/ 4$ & T shaped, straight double plate & 79 \\
\hline Hernigou and $\mathrm{Ma}^{32)}$ & 2001 & 203 & Synthetic & 120 & Case/4 & Osteotomy plate & 85 \\
\hline \multirow[t]{2}{*}{ Pornrattanamaneewong et al. ${ }^{33)}$} & 2012 & 30 & Auto & $>24$ & Comparative/3 & T buttress plate & 67 \\
\hline & 2012 & 30 & No & $>24$ & & TomoFix & \\
\hline \multirow[t]{2}{*}{ Jung et al. ${ }^{34)}$} & 2013 & 94 & Allo & 24 & Comparative/4 & Aescula plate & 67 \\
\hline & 2013 & 92 & Synthetic & 24 & & TomoFix & \\
\hline \multirow[t]{2}{*}{ Kuremsky et al. ${ }^{35)}$} & 2010 & 19 & Auto & 15 & Comparative/3 & Arthrex osteotomy plate & 72 \\
\hline & 2010 & 51 & Allo & 15 & & Arthrex osteotomy plate & \\
\hline \multirow[t]{2}{*}{ Gouin et al. ${ }^{36)}$} & 2010 & 18 & Auto & 45 & Comparative/2 & Surfix plate & 79 \\
\hline & 2010 & 22 & Synthetic & 45 & & Surfix plate & \\
\hline
\end{tabular}

CMS: Coleman methodology score, Auto: autogenous bone graft, Case: case series, Allo: allogenous bone graft, No: no filling, Synthetic: synthetic material filling, Comparative: comparative study, RCT: randomized controlled study.

\section{1) Radiological bone union period}

Only three studies with no-filling method, one study with allogenous graft and one study with synthetic graft presented mean radiological bone union periods and statistical deviations, rendering any statistical analysis meaningless. The radiological outcomes and radiological bone union periods are described in Table 7 and plotted in Fig. 2. Note that allogenous graft groups required a longer period for union.
2) Nonunion and delayed union.

Table 6 lists all papers that were included in this meta-analysis. Kolb et al. ${ }^{20)}$ defined delayed union as prolonged osteotomy healing of more than four months, and defined nonunion as no evidence of healing six months after osteotomy. Shim et al. ${ }^{22)}$ defined delayed union as the lack of bridging callus and the presence of radiolucent areas within the opening wedge defect more than three months after surgery, and nonunion as no evidence of 
Table 5. Overall Coleman Methodology Score for Each Criterion

\begin{tabular}{lllc}
\hline \multicolumn{1}{c}{ Criteria (maximum score) } & Mean & $\begin{array}{c}\text { Standard } \\
\text { deviation }\end{array}$ & Range \\
\hline Part A & & & \\
$\quad$ Study size (10) & 6.84 & 2.76 & $4-10$ \\
Mean follow-up (5) & 4.4 & 1.22 & $2-5$ \\
No. of procedures (10) & 10 & 0 & 10 \\
Type of study (15) & 7.8 & 4.58 & $0-15$ \\
Diagnostic certainty (5) & 5 & 0 & 5 \\
Surgery description (5) & 4.4 & 1.22 & $3-5$ \\
Rehabilitation description (10) & 7.6 & 4.35 & $0-10$ \\
Part B & & & \\
Outcome criteria (10) & 7.96 & 1.67 & $4-10$ \\
Procedure for outcomes (15) & 8 & 2.41 & $3-12$ \\
Selection process (15) & 15 & 0 & 15 \\
Total score (100) & 77 & 6.58 & $61-85$ \\
\hline
\end{tabular}

healing within six months. Hernigou and $\mathrm{Ma}^{32)}$ defined delayed union as the presence of pain at the osteotomy site after 45 days with evidence of loss of correction. Yacobucci and Cocking ${ }^{7}$, defining nonunion as the absence of radiological union requiring iliac crest bone grafting, reported two cases of nonunion in corrections of 15 degrees and an unrecognized fracture of the lateral hinge in one of those cases.

Nonunion and delayed union rates assessed according to the filler type are presented in Table 8. There was a similar tendency for nonunion and delayed union rates among studies classified according to the filler type. The nonunion and delayed union rates were also analyzed according to the type of fixation. Table 9 shows nonunion and delayed union rates assessed according to the fixation type. There was also a similar tendency for nonunion and delayed union rates among studies classified according to the fixation type. No series were reported on OWHTO using an

Table 6. Overall Results of Studies

\begin{tabular}{|c|c|c|c|c|c|c|c|c|}
\hline Author & Filling method & $\begin{array}{l}\text { Radiological } \\
\text { union (day), } \\
\text { mean (SD) }\end{array}$ & $\begin{array}{l}\text { Osteotomy } \\
\text { size }\left(\mathrm{mm}^{\circ}{ }^{\circ}\right) \text {, } \\
\text { mean }(\mathrm{SD})\end{array}$ & $\begin{array}{l}\text { Loss of } \\
\text { correction }\end{array}$ & Nonunion & $\begin{array}{l}\text { Delayed } \\
\text { union }\end{array}$ & $\begin{array}{l}\text { FWB } \\
\text { (wk) }\end{array}$ & $\begin{array}{l}\text { PWB } \\
(w k)\end{array}$ \\
\hline Chae et al. ${ }^{5)}$ & Tricortical iliac crest & N/A & N/A & $6 / 138$ & 0 & N/A & 6 & 2 \\
\hline Yacobucci and Cocking ${ }^{7)}$ & $\begin{array}{l}\text { Proximal tibial cortico- } \\
\text { cancellous wedge allo- } \\
\text { graft }\end{array}$ & $120(28)$ & $10.1^{\circ}$ & $1 / 50$ & $2 / 50$ & N/A & N/A & 8 \\
\hline \multirow[t]{2}{*}{ Zorzi et al. ${ }^{10)}$} & $\begin{array}{l}\text { Cancellous iliac crest } \\
\text { autologous bone }\end{array}$ & 87 & 9.9 & $1 / 23$ & 0 & N/A & N/A & 8 \\
\hline & No & 96 & 10.2 & $2 / 23$ & 0 & N/A & N/A & 8 \\
\hline Bode et al. ${ }^{15)}$ & No & N/A & N/A & 0 & $2 / 52$ & $2 / 52$ & 4 & $2-3$ \\
\hline Schroter et al. ${ }^{16)}$ & No & N/A & $8.0(2.0)$ & $2 / 35$ & N/A & N/A & $6-8$ & N/A \\
\hline El-Azab et al. ${ }^{17)}$ & No & N/A & $7.1^{\circ}\left(2.4^{\circ}\right)$ & N/A & 0 & $3 / 50$ & 6 & POD 1 day \\
\hline Brosset et al. ${ }^{18)}$ & No & $135(49)$ & $\mathrm{N} / \mathrm{A}$ & 0 & 0 & $2 / 51$ & N/A & $\mathrm{N} / \mathrm{A}$ \\
\hline El-Assal et al. ${ }^{19)}$ & No & $90(15)$ & $10.5(1.5)$ & $1 / 59$ & 0 & 0 & N/A & N/A \\
\hline Kolb et al. ${ }^{20)}$ & No & $90(14)$ & $10.9(1.5)$ & 0 & $1 / 51$ & 0 & 6 & N/A \\
\hline Zaki and $\mathrm{Rae}^{21)}$ & No & N/A & N/A & 0 & 0 & N/A & N/A & N/A \\
\hline Shim et al. ${ }^{22)}$ & Tricortical iliac crest & 90 & 6.42 & N/A & 0 & $1 / 37$ & N/A & N/A \\
\hline Schroter et al. ${ }^{23)}$ & Iliac crest bone wedge & N/A & $8.2(4.9)$ & N/A & N/A & N/A & $6-8$ & N/A \\
\hline Noyes et al. ${ }^{24)}$ & Tricortical iliac crest & 90 & $9.7(3.1)$ & $1 / 55$ & 0 & $3 / 55$ & 8 & 4 \\
\hline Haviv et al. ${ }^{25)}$ & Tricortical iliac crest & 180 & $5.0-17.5$ & 0 & 0 & 0 & 13 & 6 \\
\hline Santic et al. ${ }^{26)}$ & Femoral head & 168 & $8.6(2.0)$ & 0 & 0 & 0 & 12 & 6 \\
\hline DeMeo et al. ${ }^{27)}$ & $\begin{array}{l}\text { Tricortical iliac crest }+ \\
\text { cancellous bone chips }\end{array}$ & N/A & N/A & 0 & 0 & $1 / 20$ & 11.4 & $6-8$ \\
\hline Saito et al. ${ }^{28)}$ & $\begin{array}{l}\text { Hydroxyapatite } \\
\text { Or } \beta \text { triscalcium phos- } \\
\text { phate wedge }\end{array}$ & N/A & 12 & 0 & 0 & $1 / 64$ & N/A & N/A \\
\hline
\end{tabular}


Table 6. Continued

\begin{tabular}{|c|c|c|c|c|c|c|c|c|}
\hline Author & Filling method & $\begin{array}{c}\text { Radiological } \\
\text { union (day), } \\
\text { mean (SD) }\end{array}$ & $\begin{array}{c}\text { Osteotomy } \\
\text { size }\left(\mathrm{mm}^{\circ}{ }^{\circ}\right), \\
\text { mean }(\mathrm{SD})\end{array}$ & $\begin{array}{c}\text { Loss of } \\
\text { correction }\end{array}$ & Nonunion & $\begin{array}{l}\text { Delayed } \\
\text { union }\end{array}$ & $\begin{array}{l}\text { FWB } \\
(w k)\end{array}$ & $\begin{array}{l}\text { PWB } \\
(\text { wk) }\end{array}$ \\
\hline Saragaglia et al. ${ }^{29)}$ & $\begin{array}{l}\beta \text { triscalcium phos- } \\
\text { phate wedge }\end{array}$ & N/A & N/A & $1 / 124$ & 0 & $7 / 124$ & N/A & N/A \\
\hline Ozalay et al. ${ }^{30)}$ & $\begin{array}{l}\text { Biphasic calcium phos- } \\
\text { phate }\end{array}$ & N/A & 12.6 & 0 & 0 & N/A & N/A & N/A \\
\hline Koshino et al. ${ }^{31)}$ & $\begin{array}{l}\text { Porous hyproxyapatite } \\
\text { wedge }\end{array}$ & $96(7)$ & N/A & 0 & 0 & N/A & 8 & $3-4$ \\
\hline Hernigou and $\mathrm{Ma}^{32)}$ & Synthetic material & 90 & N/A & N/A & $1 / 203$ & $2 / 203$ & N/A & N/A \\
\hline \multirow[t]{2}{*}{$\begin{array}{l}\text { Pornrattanamaneewong } \\
\text { et al. }\end{array}$} & $\begin{array}{l}\text { Tricortical iliac crest } \\
\text { autologous bone }\end{array}$ & N/A & N/A & N/A & 0 & 0 & 4 & 2 \\
\hline & No & N/A & N/A & N/A & 0 & 0 & 4 & 2 \\
\hline \multirow[t]{2}{*}{ Jung et al. ${ }^{34)}$} & $\begin{array}{l}\text { Allogenic cancellous } \\
\text { bone chip graft }\end{array}$ & N/A & N/A & $12 / 94$ & $1 / 94$ & 0 & 10 & 6 \\
\hline & $\begin{array}{c}\text { Porous } \beta \text {-tricalcium } \\
\text { phosphate wedge }\end{array}$ & N/A & N/A & $6 / 92$ & 0 & 0 & 6 & POD 1 day \\
\hline \multirow[t]{2}{*}{ Kuremsky et al. ${ }^{35)}$} & $\begin{array}{l}\text { Tricortical iliac crest } \\
\text { autologous bone }\end{array}$ & N/A & 12.9 & N/A & 0 & N/A & N/A & 6 \\
\hline & $\begin{array}{l}\text { Freeze-dried cortico- } \\
\text { cancellous allogenic } \\
\text { structural graft }\end{array}$ & N/A & 11.8 & N/A & 0 & N/A & N/A & 6 \\
\hline \multirow[t]{2}{*}{ Gouin et al. ${ }^{36)}$} & $\begin{array}{l}\text { Iliac crest tricortical } \\
\text { autologous bone }\end{array}$ & 78 & 10.0 & $6 / 22$ & 0 & N/A & N/A & N/A \\
\hline & $\begin{array}{l}\text { Calcium-phosphate } \\
\text { cermic spacer }\end{array}$ & 154 & 10.0 & $1 / 18$ & 0 & N/A & N/A & N/A \\
\hline
\end{tabular}

SD: standard deviation, FWB: full weight-bearing, PWB: partial weight-bearing, N/A: not available, POD: postoperative day.

Table 7. Bone Union Period

\begin{tabular}{|c|c|c|}
\hline Filler type & Author & $\begin{array}{c}\text { Bone union period (day), } \\
\text { mean }(\mathrm{SD})\end{array}$ \\
\hline \multirow[t]{3}{*}{ No } & Brosset et al. $^{18)}$ & $135(49)$ \\
\hline & El-Assal et al. ${ }^{19)}$ & $90(15)$ \\
\hline & Kolb et al. ${ }^{20)}$ & $90(14)$ \\
\hline \multirow[t]{2}{*}{ Auto } & Shim et al. ${ }^{22)}$ & 90 \\
\hline & Noyes et al. ${ }^{24)}$ & 90 \\
\hline \multirow[t]{3}{*}{ Allo } & Yacobucci and Cocking ${ }^{7)}$ & $120(28)$ \\
\hline & Haviv et al. ${ }^{25)}$ & 180 \\
\hline & Santic et al. ${ }^{26)}$ & 168 \\
\hline \multirow[t]{2}{*}{ Synthetic } & Koshino et al. ${ }^{31)}$ & $96(7)$ \\
\hline & Hernigou and $\mathrm{Ma}^{32)}$ & 90 \\
\hline
\end{tabular}

SD: standard deviation, No: no filling, Auto: autogenous bone graft, Allo: allogenous bone graft, Synthetic: synthetic material filling. autologous bone graft with a locking plate.

\section{3) Loss of correction}

Yacobucci and Cocking ${ }^{7}$ reported one case of correction loss with lateral cortex fracture, wherein the correction angle was 15 degrees. Saragaglia et al. ${ }^{29)}$ also reported one case of correction loss associated with screw breakage. Tables 9 and 10 list the incidence and rate of loss of correction. There was a similar tendency for the correction loss rate when compared according to the filler type and fixation method.

\section{Comparative Studies}

Only one randomized prospective study was identified. Zorzi et al. ${ }^{10)}$ reported there was no significant difference in bone union period based on the review of a two-year comparison of 23 cases of OWHTO using autografts and another 23 cases of OWHTO without filling. As for comparative studies, there were four. Pornarattanamaneewong et al. ${ }^{33)}$ reported that there were 
no significant differences of bone union period between their groups. Kuremsky et al. ${ }^{35)}$ found that there was a 6-fold higher failure rate for the allograft group compared with the autograft group. Gouin et al. ${ }^{36)}$, comparing an autologous bone graft group with a synthetic-material graft $(\mathrm{BMCaPh})$ group, determined that correction loss was more frequent in the latter group $(5 \%$ vs. 27\%). Specifically, the presence of lateral cortical hinge tears significantly increased the risk of correction loss in the $\mathrm{BMCaPh}$ group. These results indicated that the synthetic material was less tolerant to high mechanical stress, and that radiological union occurred significantly later in the $\mathrm{BMCaPh}$ group than in the autologous bone graft group. Jung et al. ${ }^{34)}$ defined loss of correction as less than 0 degrees of mechanical tibiofemoral angle at

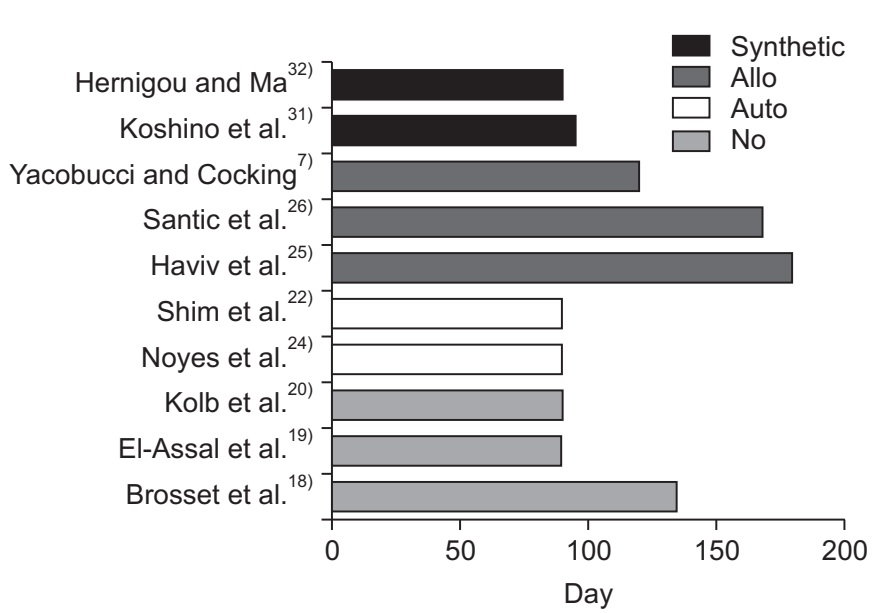

Fig. 2. Bone union period. No: no filling, Auto: autogenous bone graft, Allo: allogenous bone graft, Synthetic: synthetic material filling.
Table 8. Rates of Nonunion/Delayed Union by Filler Type

\begin{tabular}{|c|c|c|c|c|c|c|c|}
\hline Filler type & Author & $\begin{array}{l}\text { Non- } \\
\text { union }\end{array}$ & $\begin{array}{l}\text { Event } \\
\text { rate }\end{array}$ & $\begin{array}{l}\mathrm{I}_{2} \\
(\%)\end{array}$ & $\begin{array}{l}\text { Delayed } \\
\text { union }\end{array}$ & $\begin{array}{l}\text { Event } \\
\text { rate }\end{array}$ & $\begin{array}{c}\mathrm{I}_{2} \\
(\%)\end{array}$ \\
\hline \multirow[t]{7}{*}{ No filling } & & $3 / 313$ & 0.018 & 0.0 & $7 / 263$ & 0.03 & 0.0 \\
\hline & Bode et al. ${ }^{15)}$ & $2 / 52$ & 0.038 & & $2 / 52$ & 0.04 & \\
\hline & El-Azab et al. ${ }^{17)}$ & $0 / 50$ & 0.010 & & $3 / 50$ & 0.06 & \\
\hline & Brosset et al. ${ }^{18)}$ & $0 / 51$ & 0.008 & & $2 / 51$ & 0.04 & \\
\hline & El-Assal et al. ${ }^{19)}$ & $0 / 59$ & 0.020 & & $0 / 59$ & 0.01 & \\
\hline & Kolb et al. ${ }^{20)}$ & $1 / 51$ & 0.010 & & $0 / 51$ & 0.01 & \\
\hline & Zaki and $\mathrm{Rae}^{21)}$ & $0 / 50$ & 0.023 & & N/A & N/A & \\
\hline \multirow[t]{4}{*}{ Auto } & & $0 / 230$ & 0.005 & 0.0 & $4 / 92$ & 0.04 & 0.0 \\
\hline & Chae et al. ${ }^{5)}$ & $0 / 138$ & 0.004 & & N/A & N/A & \\
\hline & Shim et al. ${ }^{22)}$ & $0 / 37$ & 0.013 & & $1 / 37$ & 0.03 & \\
\hline & Noyes et al. ${ }^{24)}$ & $0 / 55$ & 0.009 & & $3 / 55$ & 0.05 & \\
\hline \multirow[t]{5}{*}{ Allo } & & $2 / 402$ & 0.004 & 0.0 & $1 / 352$ & 0.00 & 0.0 \\
\hline & $\begin{array}{l}\text { Yacobucci and } \\
\text { Cocking }^{7)}\end{array}$ & $2 / 50$ & 0.040 & & N/A & N/A & \\
\hline & Haviv et al. ${ }^{25)}$ & $0 / 22$ & 0.022 & & $0 / 22$ & 0.02 & \\
\hline & Santic et al. ${ }^{26)}$ & $0 / 310$ & 0.002 & & $0 / 310$ & 0.00 & \\
\hline & DeMeo et al. ${ }^{27)}$ & $0 / 20$ & 0.024 & & $1 / 20$ & 0.05 & \\
\hline \multirow[t]{6}{*}{ Synthetic } & & $1 / 427$ & 0.013 & 0.0 & $10 / 427$ & 0.02 & 0.0 \\
\hline & Saito et al. ${ }^{28)}$ & $0 / 64$ & 0.004 & & $1 / 64$ & 0.02 & \\
\hline & Saragaglia et al. ${ }^{29)}$ & $0 / 124$ & 0.031 & & $7 / 124$ & 0.06 & \\
\hline & Ozalay et al. ${ }^{30)}$ & $0 / 15$ & 0.005 & & $0 / 15$ & 0.03 & \\
\hline & Koshino et al. ${ }^{31)}$ & $0 / 21$ & 0.008 & & $0 / 21$ & 0.02 & \\
\hline & $\begin{array}{l}\text { Hernigou and } \\
\mathrm{Ma}^{32)}\end{array}$ & $1 / 203$ & 0.009 & & $2 / 203$ & 0.01 & \\
\hline
\end{tabular}

N/A: not available, Auto: autogenous bone graft, Allo: allogenous bone graft, Synthetic: synthetic material filling.

Table 9. Nonunion/ Delayed Union/ Correction Loss for MOWHTO with Plate Fixation

\begin{tabular}{|c|c|c|c|c|c|c|c|c|c|}
\hline Fixation type & Nonunion & Event rate & $\mathrm{I}_{2}(\%)$ & Delayed union & Event rate & $\mathrm{I}_{2}(\%)$ & Correction loss & Event rate & $\mathrm{I}_{2}(\%)$ \\
\hline Non-locking plate & $2 / 576$ & 0.02 & 0 & $15 / 388$ & 0.04 & 0 & $13 / 524$ & 0.02 & 0 \\
\hline No filling & 0/109 & 0.02 & 0 & $3 / 109$ & 0.03 & 11 & $4 / 94$ & 0.04 & 25.8 \\
\hline Auto & $0 / 230$ & 0.01 & 0 & $4 / 92$ & 0.04 & 0 & $7 / 193$ & 0.04 & 0 \\
\hline Allo & $2 / 92$ & 0.03 & 0 & $1 / 42$ & 0.04 & 0 & $1 / 92$ & 0.02 & 0 \\
\hline Synthetic & $0 / 145$ & 0.03 & $0^{\mathrm{a})}$ & $7 / 145$ & 0.05 & 0 & $1 / 145$ & 0.01 & 0 \\
\hline Locking plate & $3 / 578$ & 0.00 & 0 & $5 / 528$ & 0.01 & 0 & $0 / 526$ & 0.00 & 0 \\
\hline No filling & $3 / 204$ & 0.02 & 0 & $4 / 154$ & 0.03 & 0 & $0 / 152$ & 0.01 & 0 \\
\hline Auto & N/A & N/A & N/A & N/A & N/A & N/A & N/A & N/A & N/A \\
\hline Allo & $0 / 310$ & 0.00 & $0^{a)}$ & $0 / 310$ & 0.00 & $0^{a)}$ & $0 / 310$ & 0.00 & $0^{a)}$ \\
\hline Synthetic & $0 / 64$ & 0.00 & 0 & $1 / 64$ & 0.02 & $0^{\text {a) }}$ & $0 / 64$ & 0.01 & $0^{\text {a) }}$ \\
\hline
\end{tabular}

MOWHTO: medial opening wedge high tibial osteotomy, N/A: not available, Auto: autogenous bone graft, Allo: allogenous bone graft, Synthetic: synthetic material filling, $\mathrm{I}_{2}$ : heterogeneity.

${ }^{a)}$ Only one case series. 
Table 10. Rates of Correction Loss by Filler Type

\begin{tabular}{|c|c|c|c|c|c|}
\hline Filler type & Author & $\begin{array}{c}\text { Loss of } \\
\text { correction }\end{array}$ & Event rate & $\mathrm{I}_{2}(\%)$ & Treatment \\
\hline \multirow[t]{9}{*}{ No filling } & & $4 / 246$ & 0.02 & 0.0 & \\
\hline & Schroter et al. ${ }^{16)}$ & $3 / 35$ & 0.09 & & Two-medial dislocation of tibial plateau \\
\hline & & & & & Fixation with TomoFix and iliac creset autologous bone wedge \\
\hline & & & & & No correlation with osteotomy size \\
\hline & Brosset et al. ${ }^{18)}$ & $0 / 51$ & 0.01 & & \\
\hline & El-Assal et al. ${ }^{19)}$ & $1 / 59$ & 0.02 & & Partial loss of correction (4 degrees) \\
\hline & & & & & No intervention \\
\hline & Kolb et al. ${ }^{20)}$ & $0 / 51$ & 0.01 & & \\
\hline & Zaki et al. $^{21)}$ & $0 / 50$ & 0.01 & & \\
\hline \multirow[t]{3}{*}{ Auto } & & $7 / 193$ & 0.04 & 0.0 & \\
\hline & Chae et al. ${ }^{5)}$ & $6 / 138$ & 0.04 & & No intervention \\
\hline & Noyes et al. ${ }^{24)}$ & $1 / 55$ & 0.02 & & Revision \\
\hline \multirow[t]{6}{*}{ Allo } & & $1 / 402$ & 0.00 & 0.0 & \\
\hline & Yacobucci and Cocking ${ }^{7)}$ & $1 / 50$ & 0.02 & & Correlation with nonunion and lateral hinge fracture \\
\hline & & & & & Revision with Puddu plate and iliac crest autologous graft \\
\hline & Haviv et al. ${ }^{25)}$ & $0 / 22$ & 0.02 & & \\
\hline & Santic et al. ${ }^{26)}$ & $0 / 310$ & 0.00 & & \\
\hline & DeMeo et al. ${ }^{27)}$ & $0 / 20$ & 0.02 & & \\
\hline \multirow[t]{5}{*}{ Synthetic } & & $1 / 224$ & 0.01 & 0.0 & \\
\hline & Saito et al. ${ }^{28)}$ & $0 / 64$ & 0.01 & & \\
\hline & Saragaglia et al. ${ }^{29)}$ & $1 / 124$ & 0.01 & & $\begin{array}{l}\text { New osteotomy at the femoral level to correct the deformity } \\
2 \text { years later }\end{array}$ \\
\hline & Ozalay et al. & $0 / 15$ & 0.03 & & \\
\hline & Koshino et al. $^{31)}$ & $0 / 21$ & 0.02 & & \\
\hline
\end{tabular}

Auto: autogenous bone graft, Allo: allogenous bone graft, Synthetic: synthetic material filling.

the latest follow-up in the absence of an initial under-correction. The rate of correction loss or varus recurrence was higher in the group receiving the Asecula plate (B. Braun Korea, Seoul, South Korea) with the $\beta$-tricalcium phosphate wedge. The outcomes of all of the comparative studies are documented in Table 11. There was no significant difference in bone union period between the autogenous graft groups and no filling groups (Fig. 3).

\section{Starting Point of Weight-Bearing}

All twenty studies included in this meta-analysis reported on the starting point of weight-bearing (Table 6). There was a tendency of delayed onset of weight-bearing in patients with allograft. Jung et al. ${ }^{34)}$ permitted full weight-bearing walking one month after surgery to their allograft patients. Pornrattanamaneewong et al. ${ }^{33)}$ allowed full weight-bearing walking at the same time point for both groups (tricortical iliac crest autologous bone graft group and no filling group), and Zorzi et al. ${ }^{10}$ allowed partial weight-bearing walking at the same time point for both groups (cancellous iliac crest autologous bone graft group and no filling group); it must be noted, however, that the time point was not the endpoint of full weight-bearing walking but rather was the starting point of their rehabilitation protocol.

\section{Bone Graft-Related Problem}

Pornarattanamaneewong et al. ${ }^{33)}$ and Kuremsky et al. ${ }^{35)}$ described the graft harvest site as the source of pain that prolonged patient recovery time. Chae et al. ${ }^{5)}$ also reported the graft harvest site as the source of pain for patients in the early postoperative period especially during trunk flexion. In addition, three of their patients underwent linear fracture of the iliac bone, which was treated conservatively. Schroter et al. ${ }^{23)}$ reported that six patients suffered from hyperaesthesia or dysaesthesia at the iliac crest. Gouin et al. ${ }^{36)}$, Koshino et al. ${ }^{31)}$ and Ozalay et al. ${ }^{30)}$ all reported 
Table 11. Comparative Studies

\begin{tabular}{|c|c|c|c|c|c|c|c|c|}
\hline Study & Bone graft & $\begin{array}{l}\text { Bone union } \\
\text { period } \\
\text { (day) }\end{array}$ & $\mathrm{p}$-value & $\begin{array}{l}\text { Opening } \\
\text { size }(\mathrm{mm})\end{array}$ & Nonunion & $\begin{array}{l}\text { Delayed } \\
\text { union }\end{array}$ & $\begin{array}{l}\text { Loss of } \\
\text { correction }\end{array}$ & Fixation type \\
\hline \multirow[t]{2}{*}{ Zorzi et al. $^{10)}$} & Auto & 86.7 & 0.128 & 9.9 & 0 & N/A & $1 / 23$ & Nonlocking (Puddu) plate \\
\hline & No & 95.7 & & 10.2 & 0 & N/A & $2 / 23$ & Nonlocking (Puddu) plate \\
\hline \multirow{2}{*}{$\begin{array}{l}\text { Pornrattanamaneewong } \\
\text { et al. }^{33)}\end{array}$} & Auto & 66.5 & 0.125 & N/A & 0 & 0 & N/A & Nonlocking (T buttress) plate \\
\hline & No & 72.1 & & N/A & 0 & 0 & N/A & Locking (TomoFix) plate \\
\hline \multirow[t]{2}{*}{ Jung et al. ${ }^{34)}$} & Allo & N/A & & $\mathrm{N} / \mathrm{A}$ & $1 / 94$ & 0 & $\mathrm{~N} / \mathrm{A}$ & Nonlocking (Aescula) plate \\
\hline & Synthetic & N/A & & N/A & 0 & 0 & $\mathrm{~N} / \mathrm{A}$ & Locking (TomoFix) plate \\
\hline \multirow[t]{2}{*}{ Kuremsky et al. ${ }^{35)}$} & Auto & N/A & N/A & 12.9 & 0 & N/A & N/A & $\begin{array}{l}\text { Nonlocking (Arthrex opening wedge } \\
\text { osteotomy plate prototype set) }\end{array}$ \\
\hline & Allo & N/A & N/A & 11.8 & 0 & $\mathrm{~N} / \mathrm{A}$ & N/A & $\begin{array}{l}\text { Nonlocking (Arthrex opening wedge } \\
\text { osteotomy plate prototype set) }\end{array}$ \\
\hline \multirow[t]{2}{*}{ Gouin et al. ${ }^{36)}$} & Auto & 78 & 0.001 & 10 & 0 & N/A & $1 / 18$ & Locking (Surfix) plate \\
\hline & Synthetic & 154 & & 10 & 0 & N/A & $6 / 22$ & Locking (Surfix) plate \\
\hline
\end{tabular}

Auto: autogenous bone graft, N/A: not available, No: no filling, Allo: allogenous bone graft, Synthetic: synthetic material filling.

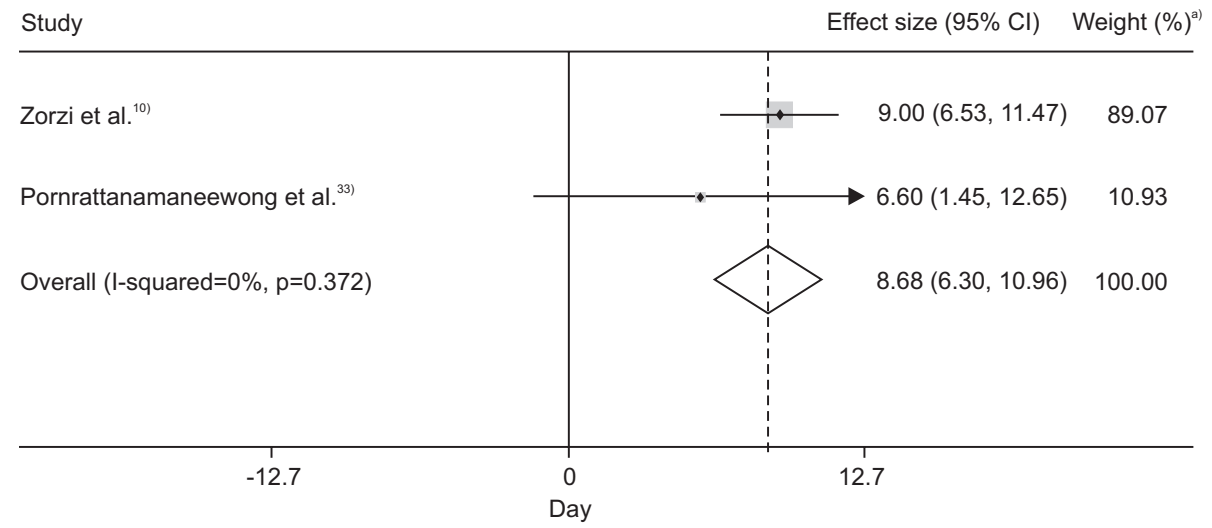

Fig. 3. Bone union period of autogenous bone graft group and no filling group. Forest plot of mean difference and $95 \%$ confidence interval (CI) for constant scores among patients assigned to Auto group or no filling group for opening wedge high tibial osteotomy. Mean difference: 8.68 (day) $=$ heterogeneity $\left(\mathrm{I}_{2}\right)=0$, $\mathrm{p}=0.372$. ${ }^{\mathrm{a})}$ Weights are from random effrects analysis. poor incorporation of substitute material.

\section{Discussion}

In this meta-analysis, the RCT was found to have demonstrated similar bone union period, union rate and correction loss rate between the patients with autogenous bone graft and those without filling. One comparative study presented similar bone union period and union and delayed union rates between the autogenous graft group and the no filling group. Another comparative study showed similar union and delayed union rates between allogenous bone graft group and the synthetic material group. One comparative study reported the autogenous bone graft group showed shorter bone union period and lower correction rate compared to the synthetic material group. In analyzing the case series, there was a similar tendency for nonunion, delayed union or correction loss rates among the groups classified according to the filler type. The bone union period showed a tendency to increase in the allogenous bone graft groups. The results of case series showed a similar tendency for union and delayed union rates among groups classified according to the graft type but a different was noted with regard to the correction loss rate.

The surgical techniques for opening wedge HTO underwent many modifications with regard to augmentation with bone grafts or bone substitutes. In general, autogenous bone graft is considered as the most successful bone filling material, owing to its osteoconductive, osteoinductive and osteogenic properties. Nevertheless, autograft harvesting involves increased operative time as well as donor-site morbidity (hyper- or dysaesthesia, pain source and iliac crest fracture $)^{24)}$. Aryee et al. ${ }^{37)}$ suggested that 
iliac bone graft should be used only in high-risk patients (obese, smoker or opening gap larger than $10 \mathrm{~mm}$ ). The use of bone allograft provides only an osteoconductive benefit, though, thanks to its availability without the need for additional surgical access, potential complications associated with the harvesting procedure of autogenous bone graft can be avoided ${ }^{26)}$. However, allografts entail the risk of virus transfer ${ }^{38}$. Synthetic materials provide the osteoconductive benefit along with initial support; however several disadvantages such as soft-tissue irritation and infection have been reported ${ }^{39)}$. van Hemert et al. ${ }^{40)}$ reported synthetic bone augmentation does not aid much in primary stability and is not intended for load-bearing. Furthermore, it must be used in a mechanically stable environment; otherwise, it cannot remodel into bone and shows slow incorporation into bone ${ }^{40)}$. Seven studies in this meta-analysis noted the disadvantages of autogenous grafts and synthetic materials. There were no studies reporting any disadvantages of allografts.

The recent trend of leaving the osteotomy with no graft is supported by some authors ${ }^{3,41}$. Zorzi et al. ${ }^{10)}$ included in this metaanalysis reported that there was no significant difference in the time to bone healing between patients that had an autologous bone graft and those that did not. They suggested that bone grafts could be reserved for special situations such as larger corrections and patients with conditions that impair bone healing. In the study, the no filling group also obtained satisfactory results and showed no differences compared with the other groups. Pornrattanamaneewong et al. ${ }^{33)}$ included in this meta-analysis reported that a significantly higher percentage of osteotomies with medial defects was found in the no bone graft group and all defects remained until the time of the 2-year follow-up. Recent studies attributed such medial defects in OWHTO to low interfragmentary movement and tissue strain underneath the locking plate. They suggested that the use of dynamic locking screws in combination with early full load-bearing would increase the interfragmentary movement and tissue strain and potentially induce good bone healing underneath the plate ${ }^{42}$.

El-Assal et al ${ }^{19)}$ performed OWHTO without bone graft for the opening size of $14 \mathrm{~mm}$ in 9 cases, and the outcome was good. Kolb et al. ${ }^{20)}$ also performed OWHTO (up to $14 \mathrm{~mm}$ ) and obtained favorable results. These two studies support the contention that OWHTO gaps of up to $14 \mathrm{~mm}$ in size can be healed without graft. Staubli et al. ${ }^{2)}$ reported that the cases with opening sizes less than $15 \mathrm{~mm}$ did not have filling defects and showed good results overall. When an osteotomy size is smaller than $14 \mathrm{~mm}$ and rigid fixation and locking system are acquired, "no filling" of the osteotomy space can be a good option for OWHTO. OWHTO without bone graft is certainly very attractive particularly because it is effective for reducing the length of surgery as well as patient morbidity.

The bone-healing process is complex and requires ideal biological and mechanical environments and factors. Kuremsky et al. ${ }^{35}$ ) included in this meta-analysis reported that lateral cortex fracture and opening size play a major role in bone union and construct failure. With higher varus angles, stability is largely dependent on the intact lateral hinge, which, if lost, would lead to the loss of resistance to axial compression forces and, thereby, loss of correction. Stability plays a major role in this regard: the mechanical factors are functions of osteotomy stability, which depends on the osteotomy size, intact lateral cortex and rigid internal fixation ${ }^{43,44)}$. Miller et al. ${ }^{45)}$ detected a $58 \%$ reduction in axial stiffness and a $68 \%$ reduction in torsional stiffness in the case of lateral tibial cortex disruption. In case of lateral cortex fracture, there is instability resulting from the insufficient primary stability provided by non-angular stable wedge plates ${ }^{46}$. Spahn et al. ${ }^{47)}$ suggested that high body mass index (BMI) after weight-bearing could be the cause of correction loss. van Houten et al. ${ }^{48)}$ noted that the use of tobacco was a major risk factor for development of nonunion in patients undergoing OWHTO. Rigid fixation, opening size and the presence/absence of lateral cortex fracture all affect bone union and maintenance of correction. In cases where there is a possibility of either correction loss or nonunion caused by lateral cortex fracture or large opening size, smoking or high BMI with non-rigid fixation, additional autologous bone graft is a better choice, with all due caution, to prevent harvest-site morbidity.

\section{Limitations}

There are several limitations to the present analysis. First, most of the reviewed studies were level IV case series; only one RCT and four comparative studies were found on the topic of OWHTO. Thus, the level IV study results could not be directly compared, which may affect the reliability of the analysis results.

Second, although the number of reviewed studies was large, the study designs were different: there were one RCT, four non-randomized trial studies and twenty uncontrolled trial studies. If the results are arranged according to the study design, the number of studies per one result analysis becomes less than 6 at the most. On the assessment of publication bias, the power of the test of a meta-analysis based on the review of less than 10 studies is too low to derive definitive conclusions. In such a case, assessment for publication bias is not appropriate according to Cochrane handbook ${ }^{49}$. Accordingly, publication bias could not be assessed for our analysis.

Third, there were no precise common definitions of radiological 
union, non-union, delayed union and loss of correction. Rather, these outcomes were assessed by the authors' own subjective methods.

Fourth, there were not sufficient studies on OWHTO without bone graft. Finally, we did not include age, varus deformity angle and bone marrow density severity into the inclusion criteria. These might have affected patient selection bias.

\section{Conclusions}

This meta-analysis evaluated the effects of four established methods of augmentation for osteotomy defect in OWHTO. According to the published studies, all of the methods (autogenous bone grafting, allogenous bone grafting, synthetic material filling and no filling) appear to be appropriate operative treatment options for osteotomy defect in OWHTO.

\section{Conflict of Interest}

No potential conflict of interest relevant to this article was reported.

\section{References}

1. Niemeyer P, Koestler W, Kaehny C, Kreuz PC, Brooks CJ, Strohm PC, Helwig P, Suedkamp NP. Two-year results of open-wedge high tibial osteotomy with fixation by medial plate fixator for medial compartment arthritis with varus malalignment of the knee. Arthroscopy. 2008;24:796-804.

2. Staubli AE, De Simoni C, Babst R, Lobenhoffer P. TomoFix: a new LCP-concept for open wedge osteotomy of the medial proximal tibia: early results in 92 cases. Injury. 2003;34 Suppl 2:B55-62.

3. Lobenhoffer P, Agneskirchner JD. Improvements in surgical technique of valgus high tibial osteotomy. Knee Surg Sports Traumatol Arthrosc. 2003;11:132-8.

4. Akizuki S, Shibakawa A, Takizawa T, Yamazaki I, Horiuchi H. The long-term outcome of high tibial osteotomy: a ten- to 20-year follow-up. J Bone Joint Surg Br. 2008;90:592-6.

5. Chae DJ, Shetty GM, Wang KH, Montalban AS Jr, Kim JI, Nha KW. Early complications of medial opening wedge high tibial osteotomy using autologous tricortical iliac bone graft and T-plate fixation. Knee. 2011;18:278-84.

6. Ahlmann E, Patzakis M, Roidis N, Shepherd L, Holtom P. Comparison of anterior and posterior iliac crest bone grafts in terms of harvest-site morbidity and functional outcomes.
J Bone Joint Surg Am. 2002;84:716-20.

7. Yacobucci GN, Cocking MR. Union of medial openingwedge high tibial osteotomy using a corticocancellous proximal tibial wedge allograft. Am J Sports Med. 2008;36:713-9.

8. Lobenhoffer P, Agneskirchner J, Zoch W. Open valgus alignment osteotomy of the proximal tibia with fixation by medial plate fixator. Orthopade. 2004;33:153-60.

9. Niemeyer P, Schmal H, Hauschild O, von Heyden J, Sudkamp NP, Kostler W. Open-wedge osteotomy using an internal plate fixator in patients with medial-compartment gonarthritis and varus malalignment: 3 -year results with regard to preoperative arthroscopic and radiographic findings. Arthroscopy. 2010;26:1607-16.

10. Zorzi AR, da Silva HG, Muszkat C, Marques LC, Cliquet A Jr, de Miranda JB. Opening-wedge high tibial osteotomy with and without bone graft. Artif Organs. 2011;35:301-7.

11. Maier RV. What the surgeon of tomorrow needs to know about evidence-based surgery. Arch Surg. 2006;141:317-23.

12. Kral JG, Dixon JB, Horber FF, Rossner S, Stiles S, Torgerson JS, Sugerman HJ. Flaws in methods of evidence-based medicine may adversely affect public health directives. Surgery. 2005;137:279-84.

13. Higgins JP, Thompson SG, Deeks JJ, Altman DG. Measuring inconsistency in meta-analyses. BMJ. 2003;327:557-60.

14. Moher D, Liberati A, Tetzlaff J, Altman DG; PRISMA Group. Preferred reporting items for systematic reviews and meta-analyses: the PRISMA statement. PLoS Med. 2009;6:e1000097.

15. Bode G, von Heyden J, Pestka J, Schmal H, Salzmann G, Sudkamp N, Niemeyer P. Prospective 5-year survival rate data following open-wedge valgus high tibial osteotomy. Knee Surg Sports Traumatol Arthrosc. 2015;23:1949-55.

16. Schroter S, Gonser CE, Konstantinidis L, Helwig P, Albrecht D. High complication rate after biplanar open wedge high tibial osteotomy stabilized with a new spacer plate (Position HTO plate) without bone substitute. Arthroscopy. 2011;27:644-52.

17. El-Azab HM, Morgenstern M, Ahrens P, Schuster T, Imhoff AB, Lorenz SG. Limb alignment after open-wedge high tibial osteotomy and its effect on the clinical outcome. Orthopedics. 2011;34:e622-8.

18. Brosset T, Pasquier G, Migaud H, Gougeon F. Opening wedge high tibial osteotomy performed without filling the defect but with locking plate fixation ( $\mathrm{TomoFix}^{\mathrm{TM}}$ ) and early weight-bearing: prospective evaluation of bone union, precision and maintenance of correction in 51 cases. Orthop 
Traumatol Surg Res. 2011;97:705-11.

19. El-Assal MA, Khalifa YE, Abdel-Hamid MM, Said HG, Bakr HM. Opening-wedge high tibial osteotomy without bone graft. Knee Surg Sports Traumatol Arthrosc. 2010;18:961-6.

20. Kolb W, Guhlmann H, Windisch C, Kolb K, Koller $\mathrm{H}$, Grutzner P. Opening-wedge high tibial osteotomy with a locked low-profile plate. J Bone Joint Surg Am. 2009;91:2581-8.

21. Zaki SH, Rae PJ. High tibial valgus osteotomy using the Tomofix plate: medium-term results in young patients. Acta Orthop Belg. 2009;75:360-7.

22. Shim JS, Lee SH, Jung HJ, Lee HI. High tibial open wedge osteotomy below the tibial tubercle: clinical and radiographic results. Knee Surg Sports Traumatol Arthrosc. 2013;21:5763.

23. Schroter S, Mueller J, van Heerwaarden R, Lobenhoffer P, Stockle U, Albrecht D. Return to work and clinical outcome after open wedge HTO. Knee Surg Sports Traumatol Arthrosc. 2013;21:213-9.

24. Noyes FR, Mayfield W, Barber-Westin SD, Albright JC, Heckmann TP. Opening wedge high tibial osteotomy: an operative technique and rehabilitation program to decrease complications and promote early union and function. Am J Sports Med. 2006;34:1262-73.

25. Haviv B, Bronak S, Thein R, Kidron A, Thein R. Mid-term outcome of opening-wedge high tibial osteotomy for varus arthritic knees. Orthopedics. 2012;35:e192-6.

26. Santic V, Tudor A, Sestan B, Legovic D, Sirola L, Rakovac I. Bone allograft provides bone healing in the medial opening high tibial osteotomy. Int Orthop. 2010;34:225-9.

27. DeMeo PJ, Johnson EM, Chiang PP, Flamm AM, Miller MC. Midterm follow-up of opening-wedge high tibial osteotomy. Am J Sports Med. 2010;38:2077-84.

28. Saito T, Kumagai K, Akamatsu Y, Kobayashi H, Kusayama Y. Five- to ten-year outcome following medial openingwedge high tibial osteotomy with rigid plate fixation in combination with an artificial bone substitute. Bone Joint J. 2014;96:339-44.

29. Saragaglia D, Blaysat M, Inman D, Mercier N. Outcome of opening wedge high tibial osteotomy augmented with a Biosorb ${ }^{\circledast}$ wedge and fixed with a plate and screws in 124 patients with a mean of ten years follow-up. Int Orthop. 2011;35:1151-6.

30. Ozalay M, Sahin O, Akpinar S, Ozkoc G, Cinar M, Cesur N. Remodeling potentials of biphasic calcium phosphate granules in open wedge high tibial osteotomy. Arch Orthop
Trauma Surg. 2009;129:747-52.

31. Koshino T, Murase T, Saito T. Medial opening-wedge high tibial osteotomy with use of porous hydroxyapatite to treat medial compartment osteoarthritis of the knee. J Bone Joint Surg Am. 2003;85:78-85.

32. Hernigou P, Ma W. Open wedge tibial osteotomy with acrylic bone cement as bone substitute. Knee. 2001;8:103-10.

33. Pornrattanamaneewong C, Numkanisorn S, Chareancholvanich K, Harnroongroj T. A retrospective analysis of medial opening wedge high tibial osteotomy for varus osteoarthritic knee. Indian J Orthop. 2012;46:455-61.

34. Jung WH, Chun CW, Lee JH, Ha JH, Kim JH, Jeong JH. Comparative study of medial opening-wedge high tibial osteotomy using 2 different implants. Arthroscopy. 2013;29:1063-71.

35. Kuremsky MA, Schaller TM, Hall CC, Roehr BA, Masonis JL. Comparison of autograft vs allograft in opening-wedge high tibial osteotomy. J Arthroplasty. 2010;25:951-7.

36. Gouin F, Yaouanc F, Waast D, Melchior B, Delecrin J, Passuti N. Open wedge high tibial osteotomies: Calcium-phosphate ceramic spacer versus autologous bonegraft. Orthop Traumatol Surg Res. 2010;96:637-45.

37. Aryee S, Imhoff AB, Rose T, Tischer T. Do we need synthetic osteotomy augmentation materials for opening-wedge high tibial osteotomy. Biomaterials. 2008;29:3497-502.

38. Gaasbeek RD, Toonen HG, van Heerwaarden RJ, Buma P. Mechanism of bone incorporation of beta-TCP bone substitute in open wedge tibial osteotomy in patients. Biomaterials. 2005;26:6713-9.

39. Pornrattanamaneewong C, Harnroongroj T, Chareancholvanich $\mathrm{K}$. Loss of correction after medial opening wedge high tibial osteotomy: a comparison of locking plates without bone grafts and non-locking compression plates with bone grafts. J Med Assoc Thai. 2012;95 Suppl 9:S21-8.

40. van Hemert WL, Willems K, Anderson PG, van Heerwaarden RJ, Wymenga AB. Tricalcium phosphate granules or rigid wedge preforms in open wedge high tibial osteotomy: a radiological study with a new evaluation system. Knee. 2004;11:451-6.

41. Brinkman JM, Lobenhoffer P, Agneskirchner JD, Staubli AE, Wymenga $\mathrm{AB}$, van Heerwaarden RJ. Osteotomies around the knee: patient selection, stability of fixation and bone healing in high tibial osteotomies. J Bone Joint Surg Br. 2008;90:1548-57.

42. Roderer G, Gebhard F, Duerselen L, Ignatius A, Claes L. Delayed bone healing following high tibial osteotomy re- 
lated to increased implant stiffness in locked plating. Injury. 2014;45:1648-52.

43. Esenkaya I, Elmali N. Proximal tibia medial open-wedge osteotomy using plates with wedges: early results in 58 cases. Knee Surg Sports Traumatol Arthrosc. 2006;14:955-61.

44. Hartford JM, Hester P, Watt PM, Hamilton D, Rohmiller M, Pienkowski D. Biomechanical superiority of plate fixation for proximal tibial osteotomy. Clin Orthop Relat Res. 2003;(412):125-30.

45. Miller BS, Dorsey WO, Bryant CR, Austin JC. The effect of lateral cortex disruption and repair on the stability of the medial opening wedge high tibial osteotomy. Am J Sports Med. 2005;33:1552-7.

46. van Raaij TM, Brouwer RW, de Vlieger R, Reijman M, Verhaar JA. Opposite cortical fracture in high tibial osteotomy: lateral closing compared to the medial opening-wedge technique. Acta Orthop. 2008;79:508-14.

47. Spahn G, Kirschbaum S, Kahl E. Factors that influence high tibial osteotomy results in patients with medial gonarthritis: a score to predict the results. Osteoarthritis Cartilage. 2006;14:190-5.

48. van Houten $\mathrm{AH}$, Heesterbeek PJ, van Heerwaarden RJ, van Tienen TG, Wymenga AB. Medial open wedge high tibial osteotomy: can delayed or nonunion be predicted? Clin Orthop Relat Res. 2014;472:1217-23.

49. Higgins JPT, Green S. Recommendations on testing for funnel plot asymmetry [Internet]. London: The Cochrane Collaboration; 2011 [cited 2015 Nov 23]. Available from: http:// handbook.cochrane.org/. 